\title{
Reestimación del impacto económico de las fiestas populares de proyección turística a través de metadatos provenientes de la telefonía móvil: Calp, un ejemplo de aplicación. José Francisco Perles Ribes* \\ Universidad de Alicante (España)
}

Elena Díaz Sánchez**

Global Centre of Excellence - GTM at LUCA (España)

\begin{abstract}
Resumen: Los estudios de impacto económico se han venido basando tradicionalmente en metodologías clásicas como el análisis input-output o diferentes tipologías de multiplicadores. La irrupción de las nuevas tecnologías y la posibilidad de captación y explotación de grandes volúmenes de datos (big data) brinda nuevas posibilidades para el análisis de estos eventos. Este artículo presenta una aplicación pionera de estas metodologías, reestimando el impacto económico generado por dos fiestas populares de un destino turístico consolidado, Calp (Alicante), a través de metadatos provenientes de la telefonía móvil. Los resultados revelan que mediante estas técnicas innovadoras se afinan los impactos evitando los riesgos de sobreestimación asociados a las técnicas clásicas de análisis económico.
\end{abstract}

Palabras clave: Impacto económico; Metadatos; Telefonía móvil; Moros y Cristianos; Oktoberfest; Calp.

Reassessment of the economic impact of popular festivals of tourist projection through metadata from mobile telephony: Calp, an example of application.

Abstract: Economic impact studies have traditionally been based on classical methodologies such as input-output analysis or multipliers. The emergence of new technologies and the possibility of capturing and exploitation of big data offers new possibilities for the analysis of these events. This article presents a pioneering application of these methodologies to two popular festivals of a consolidated tourist destination, Calp (Alicante), using metadata obtained from mobile phone devices. The results reveal that through these innovative techniques the impacts are refined avoiding the risks of overestimation associated with the classical techniques.

Keywords: Economic impact studies; Metadata; Mobile phone; Moros y Cristianos; Oktoberfest; Calp.

\section{Introducción}

La economía de la cultura, entendida en un sentido amplio, es decir, incluyendo las tradiciones y conductas que caracterizan a una sociedad o colectivo, viene despertando cada vez más interés entre los investigadores, configurándose paulatinamente como una categoría más de la economía aplicada en la medida en que se demuestran nuevas relaciones bidireccionales entre cultura y desarrollo económico (Herrero-Prieto, 2010). Una preocupación generalizada de los autores centrados en este ámbito es la estimación de los impactos económicos generados por los eventos y manifestaciones artísticas y culturales, tanto en términos directos, como en efectos arrastre generados sobre otros sectores productivos.

Los esfuerzos de los investigadores se centran tanto en los eventos de gran envergadura - Juegos Olímpicos, mundiales de fútbol, grandes premios de automovilismo, macrofestivales musicales y

* Universidad de Alicante (España); E-mail: jfperles@ajcalp.es

** Global Centre of Excellence - GTM at LUCA (España); E-mail: elena.diazsanchez@telefonica.com 
culturales, etc. -, (e.g., Dwyer, Forsyth y Spurr, 2005; Fourie y Santana-Gallego, 2011; Gelan, 2003), como en eventos de pequeña escala o dimensión reducida tales como festivales musicales locales o exposiciones culturales de duración limitada (e.g. Egresi y Kara, 2014). Las fiestas populares con proyección turística son también manifestaciones culturales de pequeña escala, susceptibles de ser analizadas bajo las metodologías de impacto económico. En concreto, la fiesta de Moros y Cristianos, con amplia implantación en municipios del sureste español (provincias de Alicante y Valencia especialmente, aunque con alguna extensión a Murcia y Albacete) ha sido objeto de análisis específicos en esta materia (Krom, 2008; Morales-Yago, Martínez-Puche y Martínez-Puche, 2018; Perles-Ribes, 2006).

Como ponen de manifiesto Jackson, Houghton, Russell y Triandos (2005) las metodologías clásicas de medición del impacto económico de eventos han sido, hasta ahora, el análisis input-output, el análisis general (CGE) y el análisis coste-beneficio. Estas estimaciones basadas en métodos clásicos no están exentas de potenciales errores. Como ponen de relieve Crompton y McKay (1994) algunos estudios de impacto económico de eventos no son objetivos ni imparciales, pues no son impulsados con el objetivo de obtener el verdadero impacto económico, sino como pretexto para justificar el apoyo público a los eventos. En este sentido, las principales fuentes de error provienen del uso de multiplicadores de renta y empleo exagerados, el uso de magnitudes equivocadas (ventas brutas en vez de beneficio o valor añadido), y fallos en el cómputo y detracción de los visitantes o impactos no causados por el evento y la omisión de los costes que sobre la población local genera el propio evento, tendiendo a una sobreestimación de los beneficios generados por el evento (Herrero-Prieto, 2010:185).

Las nuevas tecnologías y en especial, el uso de técnicas de análisis big data, entendidas como "aquellas que permiten analizar sistemáticamente y extraer de conjuntos de datos (estructurados, no estructurados o semiestructurados) que o bien son demasiado grandes o son muy complejos como para poder ser abordados con los métodos tradicionales de análisis de datos" (IBM, 2019), pueden ayudar a mejorar las estimaciones de estos impactos respecto a las obtenidas mediante los métodos tradicionales, evitando su sobreestimación. Ello es así en la medida en que facilitan el conocimiento con mayor exactitud de las magnitudes de volumen (e.g. el número de turistas o visitantes) asociadas realmente al fenómeno de estudio y reducen la dependencia de las apelaciones a la memoria de las personas entrevistadas/encuestadas que se efectúan con las técnicas tradicionales. Dicha dependencia, puede suponer una fuente importante de error en la medición de algunas de las magnitudes de interés (e.g., gasto turístico total y/o detallado por partidas, etc.).

El gran volumen de datos provisto por las estructuras big data abre nuevas oportunidades para la investigación y la toma de decisiones de los gestores de destinos turísticos, permitiendo un estudio más preciso del fenómeno turístico, la adopción de decisiones más fiables y la reducción de costes y riesgos asociados a las mismas (Frederiksen, 2012; Fuchs, Höpken \& Lexhagen, 2014). En definitiva, las ventajas del big data frente a los métodos tradicionales (encuestas y entrevistas) son mayor volumen de datos, mayor variedad de los mismos, más fiables (veracidad) e información en tiempo real (velocidad) (Song y Liu, 2017). Aplicaciones de estas técnicas al análisis de eventos turísticos basadas en datos procedentes de redes sociales son las de Bassolas, Lenormand, Tugores, Gonçalves y Ramasco (2016); Birenboim, (2016) o Wood, Guerry, Silver y Lacayo (2013)

Relacionadas con el uso de técnicas big data se encuentra la captación, análisis y explotación de metadatos procedentes de la telefonía móvil. Los trabajos efectuados en este ámbito se basan mayoritariamente en el conteo del número de móviles que hay en una determinada zona en un espacio de tiempo concreto. Aunque frecuentemente la industria y los medios de comunicación califican a este tipo de análisis como big data, los autores consideran que en un trabajo de carácter científico como el presente no pueden calificarse propiamente como tales, puesto que este tipo de datos no cumple -salvo por los de veracidad y volumen- con el resto de criterios -especialmente el de variedad- que se presuponen a las técnicas de análisis big data.

Como ejemplo de aplicación de los datos provenientes de la telefonía móvil al turismo puede citarse el artículo de Leng, Noriega, Pentland, Winder, Lutz y Alonso (2016) quienes examinan la estrategia de marketing turístico de Andorra, valorando la percepción de la experiencia turística de los visitantes y evalúan los beneficios y externalidades generados por los eventos turísticos de este país mediante técnicas de análisis aplicadas a las llamadas telefónicas (anonymous call detail records -CDRs-). Estos autores sacan a relucir que debido al mayor volumen de datos recolectados y su mayor resolución espacio-temporal, el análisis de grandes volúmenes de datos procedentes de sensores o tecnología CDR mejora el conocimiento de cuestiones relacionadas con el comportamiento de los turistas, la valoración de sus experiencias y otros importantes aspectos turísticos, respecto de las metodologías clásicas basadas en encuestas o entrevistas.

En este artículo se aborda el impacto en materia de atracción turística de dos fiestas populares que se celebran de forma anual en Calp, también conocido en el ámbito turístico como Calpe, un destino 
turístico de la provincia de Alicante, mediante el conteo de teléfonos móviles existentes durante los eventos festivos. Estas fiestas son las de Moros y Cristianos y la fiesta de la cerveza, Oktoberfest que tienen lugar durante el mes de octubre y que contribuyen a desestacionalizar la temporada turística del municipio. Hasta el conocimiento de los autores, es el primer artículo de carácter científico que aborda esta cuestión del análisis de atracción turística de fiestas populares a través de este método, lo que supone un avance respecto a la literatura existente sobre la materia.

El artículo se estructura en los siguientes puntos. Tras esta introducción, en el segundo apartado se efectúa una breve descripción de estas fiestas objeto de análisis. El tercer apartado se dedica a la explicación de la metodología utilizada. En el punto cuarto se exponen los resultados obtenidos. Finalmente, en el último apartado, se exponen las principales conclusiones.

\section{Fiestas objeto de análisis y su impacto Turístico.}

La fiesta de Moros y Cristianos se celebra en Calp desde 1977 durante el tercer fin de semana - entendido este desde jueves a domingo - del mes de octubre, coincidiendo más o menos con las fiestas patronales del municipio (Perles-Ribes, 2006). En 2018 la fiesta ha tenido lugar entre el 18 y el 22 de octubre, concentrándose los actos principales el sábado 20 por la tarde -desfile o entrada mora y cristiana, como acto más vistoso- y el domingo 21, con la celebración del desembarco y el "acto del Miracle".

Perles-Ribes (2006) señalaba de estas fiestas que propician una afluencia inusual de visitantes, en una época (octubre) en la que tradicionalmente no acudían al municipio, configurándose como un elemento importante de desestacionalización. A fecha actual, los datos existentes en el Departamento de Turismo del Ayuntamiento de Calp corroboran esta afirmación, habiéndose convertido el mes de octubre, en uno de los meses de más elevada ocupación del año.

Por su parte, la Fiesta de la Cerveza u Oktoberfest se viene celebrando en Calp desde el año 1987, lo que la hace una de las fiestas de este tipo con mayor antigüedad de las que se celebran fuera de Alemania. Inicialmente concebida de forma análoga a la fiesta original de Múnich, la fiesta fue impulsada por una agrupación de residentes de alemanes en Calp - el Club Carnaval Calpe - para financiar la fiesta de Carnaval Alemán que esta entidad venía organizando desde 1982. Sin embargo, con el tiempo, la Oktoberfest se ha consolidado como un evento propio, que ha superado en envergadura y trascendencia a la fiesta de Carnaval que pretendía apoyar. En sus años de existencia la Oktoberfest ha experimentado variaciones en la entidad organizadora -desde hace varios años se encarga de su organización el Club Creativ Calpe, sucesor del Club Carnaval Calpe-; la ubicación -con la instalación de la carpa en diversas ubicaciones del núcleo urbano de Calp-, y en las fechas de celebración -si inicialmente se celebraba durante las primeras semanas de octubre, nada más finalizar la fiesta original de Múnich, para coincidir con los puentes festivos que concurren en estas fechas, desde hace varios años se viene celebrando durante la última semana de octubre, nada más finalizar las fiestas de Moros y Cristianos, a fin de potenciar su función desestacionalizadora de la temporada turística -. En su edición de 2018, la Oktoberfest se celebró entre el 24 de octubre y el 4 de noviembre 2018 en la carpa ubicada en la zona denominada Parc de la Creativitat.

Conscientes de la importancia y el atractivo turístico de ambas fiestas, desde el Ayuntamiento de Calp se ha venido apoyando su celebración a través de esfuerzos promocionales. Fruto de los cuales, en 2013 se obtuvo la Declaración de Fiesta de Interés Autonómico para la fiesta de Moros y Cristianos- estando prevista la solicitud la Fiesta de Interés Nacional en los próximos años-y se solicitó para la Oktoberfest, la declaración de Fiesta de Interés Autonómico - no obtenida finalmente, no por su impacto o interés turístico innegable, sino por su interpretación como fiesta no autóctona por parte de la Generalitat-.

Igualmente, desde el Ayuntamiento se ha impulsado el estudio y análisis de ambas fiestas, al objeto de valorar el impacto económico y turístico de las mismas. En 2004, la Agencia de Desarrollo Local elaboró un estudio de impacto económico de la fiesta de Moros y Cristianos -cuyas principales conclusiones fueron recogidas en el artículo de Perles-Ribes (2006)-, y en 2005 se efectuó un estudio de afluencia de visitantes a la Oktoberfest cuyas conclusiones no se llegaron a publicar.

El estudio de Perles-Ribes (2006) estimaba un impacto económico de la fiesta en aproximadamente 5,5 millones de euros, de los que su gran mayoría (5,0 millones de euros) eran atribuibles al gasto derivado de la atracción turística de la fiesta (Perles-Ribes, 2006:160). Este gasto turístico se basaba en una afluencia de visitantes estimada durante dos días de duración de la fiesta cifrada en 86.168 personas (43.084 personas diarias), que generarían un gasto en bienes de consumo de 2,8 millones de euros y un gasto en alojamiento de 2,1 millones euros - basados estos últimos únicamente en una estimación de visitantes alojados en hoteles y apartamentos de 30.528 durante los dos días de duración de la fiesta 
(15.265 visitantes diarios), y estimando que el resto hasta 43.084 se alojaban en vivienda propia o cedida no generando, por tanto, gasto por este concepto- (Perles-Ribes, 2006:159).

En cuanto a la Oktoberfest, se estima por el Ayuntamiento mediante fuentes previas a este trabajo una participación en la fiesta de entre 30.000 y 40.000 personas, y según se desprende de encuestas llevadas a cabo en 2005 , un $40 \%$ de estos participantes serían propios residentes locales y un $60 \%$ visitantes procedentes de otros municipios, en su mayoría, municipios cercanos a Calp, aunque también se estima que aproximadamente el 15\% de los visitantes residen habitualmente fuera de España.

Un reciente estudio de la demanda turística de Calp llevado a cabo por el Departamento de Turismo del municipio, basado en 800 encuestas (en trabajo de campo llevado a cabo entre el 27 de julio y el 9 de octubre de 2016) ha puesto de manifiesto que tanto la Oktoberfest como la fiesta de Moros y Cristianos siguen ejerciendo un importante efecto de atracción turística para el municipio. En concreto, un 40,18\% de los visitantes encuestados ha asistido alguna vez a los actos de las fiestas de Moros y Cristianos, mientras que un 37,67\% lo han hecho a la Oktoberfest (Ayuntamiento de Calp, 2016).

Los estudios anteriores han establecido la relevancia turística de ambas fiestas a través de los métodos tradicionales, encuestas y estimaciones basadas en indicadores o multiplicadores. En 2018, y tratando de dar un paso más en la medición del impacto turístico generado por las mismas, el Ayuntamiento de Calp ha impulsado un nuevo estudio, basado esta vez en datos provenientes del conteo y explotación de los teléfonos móviles existentes en la localidad durante los períodos analizados. Esto se ha hecho de la mano de un socio tecnológico, una de las operadoras telefónicas de mayor relevancia nacional siguiendo la metodología que se expone a continuación.

\section{Metodología}

La metodología seguida para el estudio de ambas fiestas es similar y se basa en la localización y características de los teléfonos móviles existentes en el municipio de Calp durante los periodos seleccionados por el Ayuntamiento. El barrido telefónico efectuado permite obtener aproximaciones a las variables de interés turístico que se señalan en la tabla 1. Hay que señalar, con relación a las mismas, que en Calp hay dos distritos censales: núcleo urbano y urbanizaciones de periferia. Además, la estancia media, calculada en función del número de pernoctaciones efectuadas por los diferentes teléfonos, se desglosa según la nacionalidad para el caso de los turistas internacionales y según por provincia y municipio en el caso de los residentes en España. Finalmente, los datos socioeconómicos relativos a los visitantes se infieren a partir del tipo de las características técnicas del teléfono móvil que poseen.

\section{Tabla 1: Variables analizadas}

\begin{tabular}{|l|l|}
\hline \multicolumn{1}{|c|}{ Variable } & \multicolumn{1}{c|}{ Descripción } \\
\hline Visitantes por origen & $\begin{array}{l}\text { Número total de visitantes únicos en base a las siguientes variables de } \\
\text { agregación: municipio, mes y semana, origen de residencia y tipo de visitante. }\end{array}$ \\
\hline Pernoctaciones & $\begin{array}{l}\text { Número de visitantes, que han pernoctado al menos una noche en un mes, en la } \\
\text { provincia en la que se ubica el municipio de análisis. El campo pernoctaciones por } \\
\text { semana determina el número de noches de pernoctación acorde al número de días } \\
\text { de la semana de análisis. }\end{array}$ \\
\hline $\begin{array}{l}\text { Visitantes por } \\
\text { distrito }\end{array}$ & $\begin{array}{l}\text { Número de visitantes únicos en el municipio por lugar de residencia (País, } \\
\text { Provincia, Municipio) segmentados por distrito censal (en Calp hay dos únicos } \\
\text { distritos, núcleo y periferia) }\end{array}$ \\
\hline $\begin{array}{l}\text { Visitantes } \\
\text { nacionales }\end{array}$ & $\begin{array}{l}\text { Número de visitantes nacionales únicos en el municipio por provincia de } \\
\text { residencia (Provincia, mes y semana y tipo de visitante) excluyendo a los } \\
\text { visitantes regionales }\end{array}$ \\
\hline $\begin{array}{l}\text { Visitantes } \\
\text { internacionales }\end{array}$ & $\begin{array}{l}\text { Número de visitantes internacionales únicos en el municipio por país de } \\
\text { residencia (País, mes y semana y tipo de visitante) }\end{array}$ \\
\hline $\begin{array}{l}\text { Perfil } \\
\text { sociodemográfico }\end{array}$ & $\begin{array}{l}\text { Número de visitantes únicos nacionales y regionales en función del municipio } \\
\text { visitado por género, edad y poder adquisitivo. }\end{array}$ \\
\hline
\end{tabular}


Cabe resaltar que todos los procesos y análisis a los que son sometidos los datos garantizan el cumplimiento de las prescripciones legales en materia de protección de datos. En concreto, el proceso general de extracción y explotación de los datos se ilustra en la tabla 2 y puede describirse de la siguiente forma.

Tabla 2: Proceso de extracción y análisis de datos.

\begin{tabular}{|l|l|}
\hline \multicolumn{1}{|c|}{ Proceso } & \multicolumn{1}{c|}{ Detalle } \\
\hline Extracción & $\begin{array}{l}\text { Se extrae la información de los sistemas de red sobre cada cliente y su } \\
\text { localización en cada área geográfica. }\end{array}$ \\
\hline Anonimización & $\begin{array}{l}\text { Se eliminan todos los datos personales y se reemplazan por un } \\
\text { identificador irreversible. }\end{array}$ \\
\hline $\begin{array}{l}\text { Generalización a la } \\
\text { población objeto de estudio. }\end{array}$ & $\begin{array}{l}\text { Se extrapolan los datos obtenidos a fin de establecer conclusiones } \\
\text { generalizables sobre la población total, y no sólo sobre los clientes de la } \\
\text { compañía telefónica encargada del trabajo de campo. }\end{array}$ \\
\hline Agregación & $\begin{array}{l}\text { Se agregan los datos para cumplir las restricciones impuestas por la } \\
\text { Agencia Española de Protección de Datos. }\end{array}$ \\
\hline
\end{tabular}

La geolocalización de los teléfonos se efectúa a través de su conexión con las estaciones base (antenas) que les prestan servicio. Estas antenas tienen asignadas unas áreas de cobertura geográfica y capturan los eventos efectuados por los teléfonos móviles en su radio de acción. A fin de efectuar el seguimiento del aparato, establecer su patrón de comportamiento y movilidad e inferir las características de su propietario (pero preservando su identidad) con relación a las variables turísticas de interés objeto de análisis, los teléfonos están vinculados a un identificador. Los eventos que se capturan por las antenas son tanto activos -realización o recepción de llamadas, envío o recepción de mensajes de texto, encendido y apagado el terminal, etc. - como pasivos, es decir, el mero desplazamiento del usuario a través del área de cobertura de las distintas antenas sin efectuar ninguna actuación.

Extraídos y anonimizados los datos, por la empresa se realiza un proceso de extrapolación que permite generalizar las conclusiones al conjunto de la población objeto de análisis. Estas extrapolaciones se basan en la cuota de mercado micro geográfica de las diversas empresas proveedoras de servicios telefónicos y en otras fuentes secundarias provenientes tanto de estudios privados de mercado, como de fuentes públicas (estudios del Centro de Investigaciones Sociológicas y datos del Instituto Nacional de Estadística).

El paso previo a la explotación de los datos es un proceso de agregación, impuesto por la Agencia Española de Protección de Datos, al objeto de garantizar la irreversibilidad de los procesos de anonimización y la identificación concreta de cualquier usuario individual con base a los datos e informes finalmente entregados al cliente. De esta forma, los datos se entregan agrupados y nunca pueden hacer referencia a grupos inferiores a quince individuos. La tecnología permite trabajar con diversos niveles de agregación en función de las características de la red de telefonía móvil disponible en el destino (barrio, distrito municipal, municipio, provincia, código postal). En el caso de Calp el nivel de agregación/ desagregación de los datos ha sido el distrito censal. Finalmente, la explotación de los datos obtenidos se efectúa mediante estadísticas descriptivas basadas en el análisis de tablas de contingencia.

En cuanto a las definiciones y detalles concretos, es de interés señalar que el proceso de análisis permite distinguir entre residentes, personas en tránsito y visitantes. Estos últimos son los que realmente interesan y se tienen en cuenta a los efectos del análisis.

Las personas en tránsito -teléfonos móviles de personas que, viajando en vehículo propio, o mediante cualquier otro medio, son captadas por las antenas - son cuantificadas y excluidas del análisis. Los residentes son igualmente excluidos. La distinción entre residentes y visitantes se efectúa con base al comportamiento experimentado por el teléfono (específicamente el lugar en el que mayoritariamente ha pernoctado el móvil) durante un período previo de seis meses al análisis efectuado. Igualmente, con base a la identificación de las pernoctaciones efectuadas durante el período de análisis, se obtiene también una clasificación de los teléfonos que se encuentran en el destino entre excursionistas (no efectúan pernoctación en el destino en el período de análisis considerado), turistas nacionales -pudiendo distinguir entre origen la misma Comunidad Autónoma o resto de España -, turistas internacionales -se identifican por estar conectados mediante roaming, comprobando el país y operador de origen y 
se descartan aquellos móviles que puedan pertenecer a residentes que tengan un segundo móvil para hablar con el país de origen del móvil o viven en zonas limítrofes -y trabajadores.

Dejando de lado los aspectos prácticos de la metodología, pero sin abandonar este plano, respecto al período analizado y su justificación, existen dos períodos diferenciados. Para las fiestas de Moros y Cristianos, como se ha dicho, en su edición de 2018, se celebraron entre el 18 y el 22 de octubre. Los datos recogidos para el estudio de esta fiesta abarcan entre el 17 y 23 de octubre. A fin de disponer de un marco comparativo (control) por parte del Ayuntamiento, se seleccionó la semana de entre el 3 y el 9 de octubre como semana de referencia. El objetivo de tener una semana de control es poder contrastar sí efectivamente la celebración de las fiestas implican un comportamiento turístico diferenciado respecto de cualquier otra semana del mes. La semana posterior a la fiesta (del 24 al 30 de octubre) se desestimó como opción de control por coincidir con la celebración de la Oktoberfest, que se entiende ejerce su propia atracción turística y supone un potencial elemento distorsionador de los resultados. La semana seleccionada como control tiene el inconveniente de coincidir con el festivo del 9 de octubre de la Comunidad Valenciana. Si bien, se prefirió esa semana a la posterior (del 10 al 17 de octubre) por entender que el potencial distorsionador de ese día festivo sería inferior al provocado por el puente del 12 de octubre coincidente en esa semana.

Para el caso de la Oktoberfert, en su edición de 2018, se celebró entre el 24 de octubre y el 4 de noviembre de 2018. La recogida de datos aquí es coincidente con el período indicado. Y como en el caso anterior, a fin de disponer de un marco comparativo (control) por parte del Ayuntamiento, se seleccionó un período entre el 3 y el 14 de octubre como referencia. La semana anterior a la fiesta (del 17 al 23 de octubre) se desestimó como opción de control por coincidir con la celebración de los Moros y Cristianos. Como antes, el período de control seleccionado tiene como inconveniente coincidir con el festivo del 9 de octubre de la Comunidad Valenciana y el 12 de octubre de ámbito nacional. Sin embargo, el período de celebración de la Oktoberfest abarcó el festivo del 1 de noviembre, por lo que se entiende puede existir un cierto equilibrio entre ambas semanas.

En cualquier caso, cabe apuntar, con relación a la posible interpretación causal de los resultados obtenidos, que aunque el contraste de los datos correspondientes a las festividades respecto a las semanas de control escogidas tratan, en principio, de facilitar dicha interpretación causal - es decir, aproximar el verdadero impacto turístico de la fiesta-, el ejercicio efectuado debe entenderse con muchas precauciones y una mera aproximación. Obtener verdaderas interpretaciones causales -es decir, interpretar el efecto de atracción que en sí, las fiestas generan-, obligaría establecer un genuino contrafactual a la celebración de las mismas, que sería observar el comportamiento turístico en las semanas de análisis si las fiestas no hubieran tenido lugar (Imbens y Rubin, 2015), lo que con datos de un único ejercicio, como los que se disponen para este trabajo, es imposible. Con datos longitudinales, sería tal vez posible efectuar la interpretación causal para la Oktoberfest, que ha ido experimentando variaciones en la fecha de celebración con mayor asiduidad que la fiesta de Moros y Cristianos. Para esta última, cuya celebración suele variar en función de la celebración de la fiesta patronal (22 de octubre) sería también factible, aunque el número de años disponibles debería ser tal que permitiera la comparación de varias ediciones de la fiesta en función de si se celebra la segunda o tercera semana octubre, coincidiendo o no con la celebración de la fiesta patronal. Por este motivo, los resultados que se detallan en el próximo apartado reflejan más la situación del destino durante las fechas de celebración de las fiestas, que no un verdadero impacto en la afluencia turística generado por las mismas.

\section{Resultados}

\subsection{Afluencia turística a la fiesta de Moros y Cristianos.}

El primer dato relevante puesto de manifiesto por el estudio efectuado es que el número de visitantes registrados en Calp entre el 17 y el 23 de octubre (la semana festiva) alcanza las 56.707 personas (tabla 3). Esta cifra es un diez por cien inferior a la registrada durante la semana de control. Igualmente, esta cifra es sustancialmente inferior a los 86.168 visitantes estimados para los dos días considerados en el estudio de impacto económico de Perles-Ribes (2006). Sin embargo, ambas cifras no son directamente comparables, pues la cifra arrojada por la empresa hace referencia a individuos que se encuentran en Calp durante la semana analizada, y son computadas una única vez, con independencia de los días que permanecen en el municipio. Es decir, un sujeto A que se haya computado por encontrarse en el municipio, por ejemplo, el lunes, ya no vuelve a incluirse en el cómputo, aunque permanezca en Calp cualquier otro día de la semana. La cifra relevante, por ser la realmente comparable, es pues la de los 
teléfonos que durante un día concreto se encuentran en Calp (figura 1), y para los dos días festivos (sábado y domingo) considerados en el estudio de Perles-Ribes (2006) esta cifra alcanza, en 2018, en realidad, 39.796 visitantes. A la vista de este resultado, el impacto turístico calculado en aquel estudio estaría sobreestimado en prácticamente el doble la atracción real de la fiesta, corrigiendo el impacto a la baja desde los 4,9 millones de euros a una cifra -valores de 2004- de 2,3 millones de euros.

\section{Figura 1: Teléfonos móviles computados en un día concreto: semana control, semana festiva Moros y Cristianos}

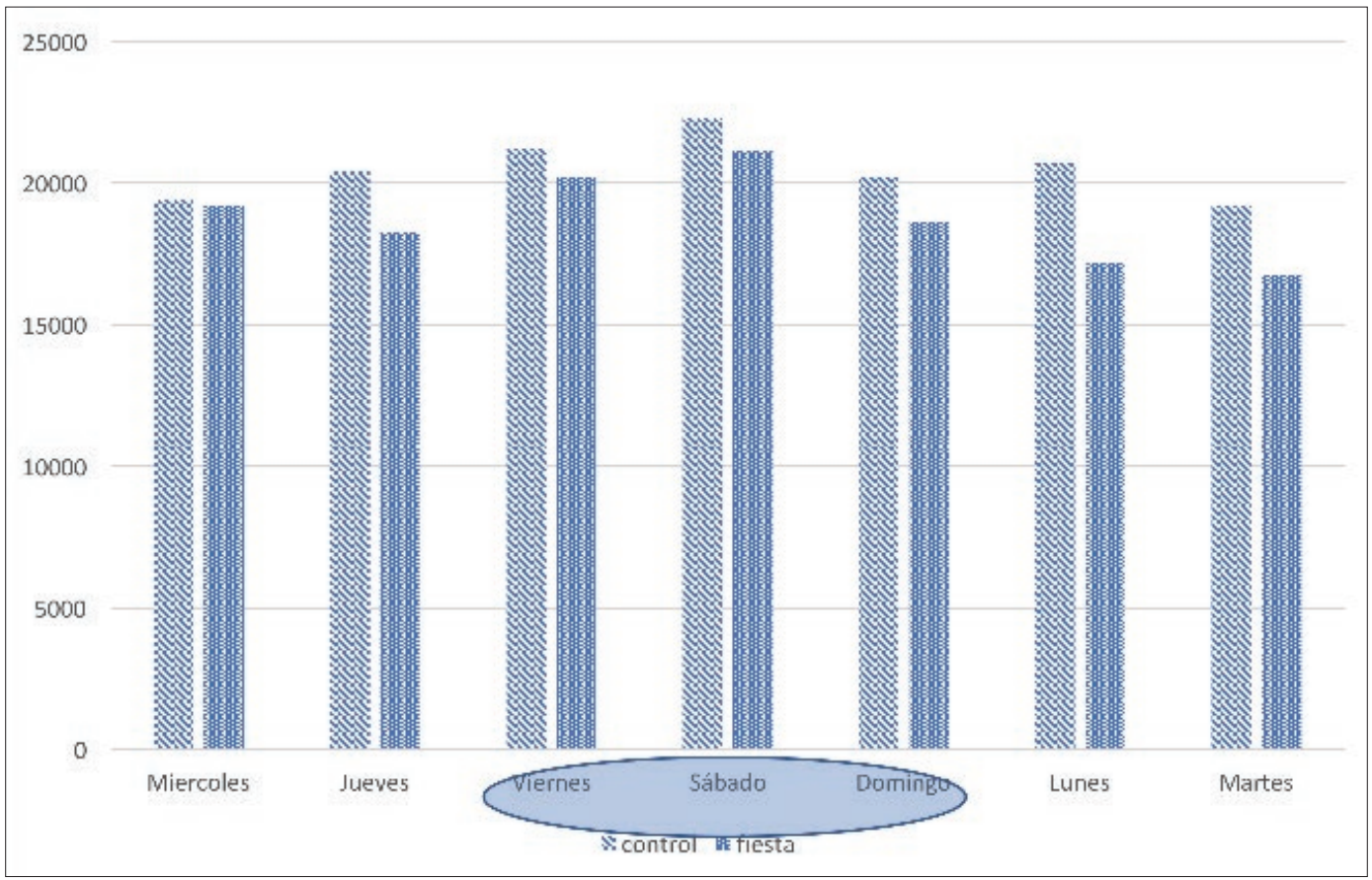

Nota: El óvalo representa los días fuertes de la fiesta, que coinciden con fin de semana. Los residentes en Calp están excluidos del análisis.

La reducción en el número de visitantes entre la semana festiva y la de control es generalizada entre todo tipo de turistas, si bien quienes más contribuyen a la misma son los turistas nacionales -de fuera de la provincia de Alicante-, que experimentan una reducción del $22 \%$, puesto que turistas internacionales o visitantes del entorno cercano experimentan a lo largo de la semana caídas mucho más reducidas cifradas en torno al 7\% (tabla 3).

Tabla 3: Visitantes únicos por nacionalidad durante la celebración de los Moros y Cristianos.

\begin{tabular}{|l|r|r|r|r|}
\hline \multicolumn{1}{|c|}{ Origen } & Moros & \multicolumn{1}{c|}{ Control } & Total general & Variación \% \\
\hline Internacional & 29.390 & 31.622 & 61.012 & $-7,06$ \\
\hline Nacional & 8.996 & 11.560 & 20.556 & $-22,18$ \\
\hline Regional & 18.321 & 19.689 & 38.010 & $-6,95$ \\
\hline Total general & 56.707 & 62.871 & 119.578 & $-9,80$ \\
\hline
\end{tabular}

Fuente: LUCA Estudio de afluencia turística fiestas de Moros y Cristianos de Calp 2018 
El hecho de que el número de visitantes global estimado durante la semana festiva no supere a la correspondiente a la semana de control, no implica obligatoriamente que la fiesta carezca de impacto turístico. Así, un resultado relevante del estudio está relacionado con el comportamiento de las pernoctaciones. Como ocurre con el número de visitantes, se observa una ligera disminución en la semana festiva respecto de la de control. Sin embargo, este comportamiento agregado esconde un notorio incremento de las pernoctaciones de un solo día en el ámbito nacional $(+2,13 \%)$ y regional $(+12,00 \%)$. Y, de mucha mayor relevancia, un incremento sustancial de las pernoctaciones de cuatro y cinco días para el ámbito internacional $(+8,65 \%$ y $+58,37 \%$ respectivamente). Este mayor número de pernoctaciones en el tramo de mayor duración es un signo inequívoco de la atracción turística, y por tanto el impacto económico, que genera esta fiesta.

Es más, si se acota el examen de la afluencia de visitantes a la zona (distrito 1), días y horarios donde tienen lugar los actos festivos (tabla 4) se observa que durante el fin de semana se concentran crecimientos de visitantes de hasta el $31,68 \%$ en la franja de tarde (15 a 21 horas) o del 20,77\% en la franja de mañana respecto a la semana de control. Este impacto positivo viene corroborado por el descenso generalizado en el número de visitantes que se produce en el distrito 2 (periferia) tanto durante la semana, como durante el fin de semana. Por tanto, resulta evidente el trasvase o concentración de turistas desde otras zonas del destino hacia el área en la que discurren los actos festivos.

Tabla 4: Visitantes únicos por distrito. Valor absoluto.

\begin{tabular}{|c|c|c|c|c|c|c|c|c|c|c|c|c|c|}
\hline \multicolumn{14}{|c|}{ Semana de control } \\
\hline \multirow[t]{3}{*}{ Distrito } & \multicolumn{6}{|c|}{1} & \multicolumn{6}{|c|}{2} & \multirow{3}{*}{ TOTAI } \\
\hline & \multicolumn{3}{|c|}{ Lunes-Jueves } & \multicolumn{3}{|c|}{ Viernes a domingo } & \multicolumn{3}{|c|}{ Lunes-Jueves } & \multicolumn{3}{|c|}{ Viernes a domingo } & \\
\hline & 9 a 15 & 15 a 21 & 21 a 9 & 9 a 15 & 15 a 21 & 21 a 9 & 9 a 15 & 15 a 21 & 21 a 9 & 9 a 15 & 15 a 21 & 21 a 9 & \\
\hline Excursionista & 5284 & 3043 & 1912 & 3880 & 2419 & 1798 & 16549 & 10054 & 8024 & 13663 & 8854 & 7204 & 82684 \\
\hline Turista & 1695 & 1219 & 2279 & 1286 & 1018 & 2086 & 5248 & 4519 & 8604 & 4192 & 3744 & 7246 & 43136 \\
\hline Total & 6979 & 4262 & 4191 & 5166 & 3437 & 3884 & 21797 & 14573 & 16628 & 17855 & 12598 & 14450 & 125820 \\
\hline \multicolumn{14}{|c|}{ Semana de moros } \\
\hline Excursionista & 5272 & 3095 & 1628 & 4508 & 3055 & 1922 & 13583 & 8565 & 6632 & 10595 & 8005 & 6306 & 73166 \\
\hline Turista & 2035 & 1369 & 2267 & 1731 & 1471 & 2446 & 5075 & 4540 & 7759 & 4275 & 3533 & 7284 & 43785 \\
\hline Total & 7307 & 4464 & 3895 & 6239 & 4526 & 4368 & 18658 & 13105 & 14391 & 14870 & 11538 & 13590 & 116951 \\
\hline
\end{tabular}

Fuente: Elaboración propia a partir de LUCA Estudio de afluencia turística fiestas de Moros y Cristianos de Calp 2018

Nota: Sombreado lugar y fecha en que tienen lugar los eventos festivos.

El examen en detalle del origen de los visitantes (tablas 5 y 6) apunta a la misma dirección. Si en el ámbito del mercado nacional, son los procedentes de Valencia y Madrid los que en mayor medida acuden a Calp durante toda la semana, experimentando ambas reducciones significativas respecto a los manifestados durante la semana de control; cuando únicamente se considera el fin de semana de los actos festivos y el lugar donde se ubica la fiesta, son los procedentes de Madrid, Valencia y Murcia los que mayor presencia presentan, experimentando aumentos notables respecto al fin de semana de control. En el ámbito internacional, por su parte, son los procedentes del Reino Unido, Alemania, Francia, Bélgica y Holanda los que mayor presencia tienen en el municipio, experimentando todos ellos reducciones respecto a la semana de control excepto por el caso de los franceses y los holandeses que muestran aumentos muy notables. 
Tabla 5: Visitantes únicos españoles por procedencia durante las fiestas de Moros y Cristianos. Distrito 1 y fin de semana.

\begin{tabular}{|l|r|r|r|}
\hline \multicolumn{1}{|c|}{$\begin{array}{c}\text { Provincia de } \\
\text { procedencia }\end{array}$} & \multicolumn{1}{|c|}{ Semana Control } & Semana Moros & \multicolumn{2}{c|}{ Variación \% } \\
\hline Valencia & 623 & 820 & 31,62 \\
\hline Madrid & 521 & 563 & 8,06 \\
\hline Murcia & 141 & 210 & 48,94 \\
\hline Cuenca & 77 & 0 & $-100,00$ \\
\hline Albacete & 71 & 61 & $-14,08$ \\
\hline Barcelona & 71 & 155 & 118,31 \\
\hline Castellón & 31 & 151 & 387,10 \\
\hline Navarra & 16 & 71 & 343,75 \\
\hline Otros & 727 & 650 & $-10,59$ \\
\hline Total & 2.278 & 2.681 & 17,69 \\
\hline
\end{tabular}

Fuente: Elaboración propia a partir de LUCA Estudio de afluencia turística fiestas de Moros y Cristianos de Calp 2018

Tabla 6: Visitantes únicos internacionales por procedencia durante las fiestas de Moros y Cristianos. Distrito 1 y fin de semana.

\begin{tabular}{|l|r|r|r|}
\hline País de procedencia & Control & Moros & \multicolumn{2}{c|}{ Variación } \\
\hline Reino Unido & 1.406 & 2.429 & 72,76 \\
\hline Bélgica & 1.391 & 1.401 & 0,72 \\
\hline Francia & 1.011 & 1.516 & 49,95 \\
\hline Alemania & 1162 & 1105 & $-4,91$ \\
\hline Países Bajos & 530 & 829 & 56,42 \\
\hline Suiza & 582 & 337 & $-42,10$ \\
\hline Federación Rusa & 352 & 234 & $-33,52$ \\
\hline Suecia & 135 & 179 & 32,59 \\
\hline Noruega & 72 & 216 & 200,00 \\
\hline Italia & 93 & 98 & 5,38 \\
\hline Irlanda & 88 & 54 & $-38,64$ \\
\hline Islandia & 35 & 92 & 162,86 \\
\hline Otros & 343 & 401 & 16,91 \\
\hline Total & 7.200 & 8.891 & 23,49 \\
\hline
\end{tabular}

Fuente: Elaboración propia a partir de LUCA Estudio de afluencia turística fiestas de Moros y Cristianos de Calp 2018

En este sentido, parece claro que la fiesta es un elemento atractor hacia esta importante zona del destino, puesto quienes no la conocen, ni quizá la conocerían en ausencia de la fiesta -quedarían alojados y restringirían su movimiento alrededor de la zona de playas-, aprovechan la fiesta para conocerla. 


\subsection{Afluencia turística de la fiesta de Oktoberfest.}

Los datos relativos a la Oktoberfest cobran si cabe más interés al suponer la primera estimación mediante métodos científicos que se efectúa sobre la misma. Los resultados obtenidos señalan que la afluencia de visitantes al municipio durante los días que dura la fiesta se cifra en 95.239 personas (tabla 7), cifra ligeramente inferior (-1,98\%) respecto al período de control elegido. Como en el caso de los Moros y Cristianos, la reducción observada obedece al comportamiento generalizado de todas las procedencias, si bien, la contribución más negativa la ejercen los turistas nacionales -de fuera de la provincia de Alicante- que experimentan una reducción del $15 \%$, pues los visitantes internacionales se reducen en un porcentaje sensiblemente inferior (-7\%) y los procedentes de municipios situados alrededor de Calp experimentan un claro aumento del $17 \%$. Por tanto, resulta evidente el efecto atractor de esta fiesta respecto a los municipios del entorno.

Tabla 7: Visitantes únicos por nacionalidad durante la Oktoberfest.

\begin{tabular}{|l|r|r|r|}
\hline \multicolumn{1}{|c|}{ Tipología } & Control & Oktoberfest & \multicolumn{2}{c|}{ Variacion } \\
\hline Internacional & 44.585 & 41.261 & $-7,46$ \\
\hline Nacional & 23.283 & 19.683 & $-15,46$ \\
\hline Regional & 29.299 & 34.295 & 17,05 \\
\hline Total general & 97.167 & 95.239 & $-1,98$ \\
\hline
\end{tabular}

Fuente: LUCA Estudio de afluencia turística fiestas de la Oktoberfest de Calp 2018

Como en el caso anterior, este dato hace referencia a visitantes únicos, con independencia de los días de permanencia en el municipio. Si en vez de contar visitantes únicos, se cuentan los visitantes que en un día concreto se encuentran en Calp (figura 2), la cifra anterior asciende notablemente, a 228.961 personas. Por tanto, si se da por buena la cifra tradicional de 30.000 visitantes a la feria previa a este trabajo, obtenida a partir de los consumos efectuados y el aforo de la carpa en la que se celebran los actos, ello significaría que aproximadamente un 13\% de los visitantes que se encuentran en Calp durante esta semana, acuden a la fiesta.

Figura 2: Teléfonos móviles computados en un día concreto: período control, período festivo Oktoberfest

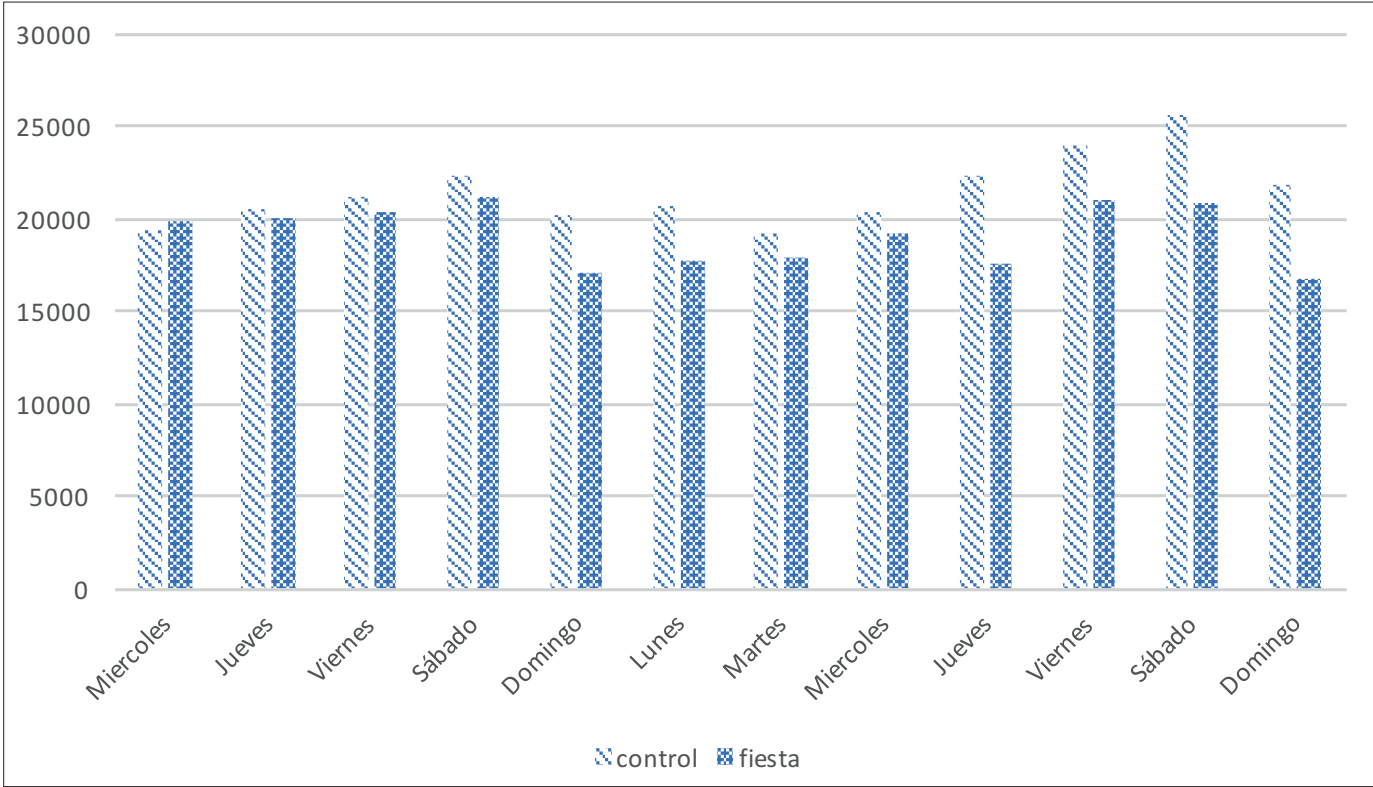

Nota: Los residentes en Calp están excluidos del análisis. 
El análisis de procedencias (tabla 8 y 9) muestra que la mayor afluencia de visitantes nacionales durante la semana festiva es concordante con los mercados tradicionales de Calp, a saber: procedentes de Valencia y Madrid, si bien ambas experimentan reducciones importantes respecto de los valores de la semana de control. Por su parte, en el ámbito internacional son los procedentes de Reino Unido, Francia, Bélgica y Alemania las mayores nacionalidades, experimentando, como en el caso de los nacionales, reducciones todas ellas excepto para el caso de Francia, que experimenta un aumento significativo, en principio, no atribuible al impacto de la Oktoberfest.

Tabla 8: Visitantes únicos españoles por procedencia durante las fiestas de la Oktoberfest. 10 principales provincias.

\begin{tabular}{|l|r|r|r|}
\hline Provincia & Control & Oktoberfest & Variación \\
\hline Valencia/València & 14.988 & 10.783 & $-28,06$ \\
\hline Madrid & 12.073 & 9.099 & $-24,63$ \\
\hline Murcia & 1.832 & 1.852 & 2,29 \\
\hline Barcelona & 982 & 1.276 & 29,94 \\
\hline Albacete & 1.255 & 730 & $-41,83$ \\
\hline Toledo & 1.083 & 808 & $-25,39$ \\
\hline Zaragoza & 954 & 545 & $-42,87$ \\
\hline Castellón/Castelló & 816 & 574 & $-29,66$ \\
\hline Bizkaia & 846 & 487 & $-42,43$ \\
\hline Gipuzkoa & 685 & 543 & $-20,73$ \\
\hline
\end{tabular}

Fuente: LUCA Estudio de afluencia turística fiestas de la Oktoberfest de Calp 2018

Tabla 9: Visitantes únicos internacionales por procedencia durante las fiestas de la Oktoberfest. 10 principales países.

\begin{tabular}{|l|r|r|r|}
\hline Nacionalidad & Control & Oktoberfest & Variacion \\
\hline Reino Unido & 22.998 & 20.582 & $-10,51$ \\
\hline Alemania & 17.703 & 11.719 & $-33,80$ \\
\hline Francia & 12.496 & 14.641 & 17,17 \\
\hline Bélgica & 13.255 & 12.983 & $-2,05$ \\
\hline Países Bajos & 7.739 & 6.810 & $-12,00$ \\
\hline Suiza & 9.184 & 3.405 & $-62,92$ \\
\hline Federación Rusa & 4.400 & 2.018 & $-54,14$ \\
\hline Noruega & 1.918 & 3.214 & 67,57 \\
\hline Suecia & 1.995 & 1.838 & $-7,87$ \\
\hline Italia & 1.147 & 856 & $-23,28$ \\
\hline
\end{tabular}

Fuente: LUCA Estudio de afluencia turística fiestas de la Oktoberfest de Calp 2018

Las pernoctaciones durante los días que se celebra la fiesta, por su parte, también experimentan, en términos globales, una reducción respecto de las registradas durante la semana de control (-12,96\%). Si bien, como en el caso anterior, esta tendencia general encubre algunos comportamientos diferenciados, como son un notorio incremento de las pernoctaciones de tres, cuatro y siete días en el ámbito de los visitantes internacionales. 
El análisis de la localización de los visitantes pone de manifiesto un menor efecto difusor de los beneficios de esta fiesta, en el sentido en que la misma se concentran en la carpa donde tienen lugar los actos y sus alrededores (distrito 2, en este caso). De hecho, los visitantes al núcleo urbano (distrito 1) experimentan una clara disminución respecto a los de la semana de control (-19,25\%), muy superior a la experimentada en el distrito 2 , que se cifra en apenas el $6 \%$.

\subsection{Interpretación de los resultados obtenidos.}

Una interpretación natural de los resultados obtenidos llevaría a la lógica conclusión de que las fiestas de Moros y Cristianos y la Oktoberfest en Calp generan un impacto turístico-económico negativo, habida cuenta que el volumen de visitantes durante los períodos festivos resultan inferiores a los existentes en los períodos de control seleccionados. Esto no es evidentemente así. Ambas fiestas generan un impacto positivo en términos de afluencia turística al destino, si bien parece evidente que el mismo es de menores dimensiones que el estimado previamente mediante las técnicas clásicas de impacto económico.

En este sentido, en el apartado relativo a la metodología ya se expusieron las reservas y precauciones con las que deben interpretarse los resultados obtenidos, especialmente si pretenden analizarse desde un punto de vista causal. Allí se comentó que los períodos de control no son auténticos contrafactuales de que ocurriría en el municipio de no celebrarse los actos festivos, sino meras aproximaciones. La selección de los períodos de control por los investigadores, efectuada en buena ciencia a priori de la captación de datos, obedece a la intención de obtener datos comparativos en este estudio respecto de los obtenidos en trabajos previos como el de Perles-Ribes (2006) en los que se utilizaba el mes de octubre como marco comparativo de referencia. Esta estrategia, se ha demostrado errónea.

En Perles-Ribes (2006) ya se puso de manifiesto que la confluencia de los actos festivos durante las dos últimas semanas de octubre ha provocado la prolongación de la temporada turística de forma significativa, haciendo de este mes uno de los de mayor ocupación del municipio, por encima incluso de lo observado durante la Semana Santa (Perles-Ribes, 2006:157). Parece que, con el tiempo, y la confluencia de otros periodos festivos como el 9 y el 12 de octubre, se ha acentuado esta tendencia haciendo al propio mes de octubre inviable como período de control para este tipo de ejercicios. En este sentido, una mejor aproximación al verdadero impacto económico de ambas fiestas se hubiera obtenido seleccionando un período de control fuera de la temporada alta -tercera o cuarta semana del mes de noviembre hubiera sido una mejor elección-. Lamentablemente, los datos para este período de temporada baja no se encuentran disponibles actualmente, y aunque ello supone una limitación importante de esta investigación, representa, a su vez, una importante lección a obtener de la misma: la tecnología, aunque favorece la obtención de resultados, no resuelve por sí misma todas las cuestiones que giran en torno a una investigación, y no alcanzan todo su potencial si no van acompañadas de un diseño de investigación adecuado al fenómeno que se pretende estudiar. Aunque únicamente fuera por este motivo, los autores entienden que el ejercicio efectuado ya valdría la pena.

\section{Conclusiones}

Las manifestaciones culturales, entre ellas, las festivas, son un importante atractivo turístico y una oportunidad para la desestacionalización y mejoras de la competitividad de muchos destinos. Tradicionalmente, el impacto económico de estos eventos ha sido estimado mediante técnicas clásicas de análisis económico como el análisis input-output o los multiplicadores y estudios basados en encuestas. Sin embargo, el avance operado por la tecnología y su implantación masiva en el marco de lo que vienen a denominarse destinos turísticos inteligentes (Fernández-Alcantud, López-Morales, Moreno-Izquierdo, Perles-Ribes, Ramón-Rodríguez y Such-Devesa, 2017; Perles-Ribes, Ramón-Rodríguez, Vera-Rebollo y Ivars-Baidal, 2017; Perles-Ribes y Ivars-Baidal, 2018), está permitiendo el análisis de grandes volúmenes de datos procedentes de sensorización, redes sociales o redes de telefonía móvil. Esto brinda nuevas oportunidades para el análisis de impacto económico, pues permite afinar mejor los datos y supuestos de partida en términos de volumen de visitantes que efectivamente acuden a un evento, y sus principales características.

El presente artículo ha abordado el estudio de la afluencia turística a dos manifestaciones festivas populares -la fiesta de Moros y Cristianos y la fiesta de la Cerveza u Oktoberfest- que tienen lugar durante el mes de octubre en Calp, un destino consolidado de la provincia de Alicante. Ello ha permitido estimar, por primera vez con métodos científicos la afluencia turística de la Oktoberfest y matizar el 
impacto económico de la fiesta de Moros y Cristianos que fue objeto de un estudio previo mediante las técnicas clásicas de análisis.

Los resultados han puesto de manifiesto, en el ámbito local, tres aspectos básicos. En primer lugar, que ambas fiestas son, efectivamente, un importante motor de atracción turística del destino, confirmando el resultado general obtenido en Perles-Ribes (2006). Si se considera que la temporada turística tradicional en el municipio abarcaba hasta la primera quincena de octubre, los festivos que concurren durante el mes contribuyen a la desestacionalización, alargando la misma una quincena adicional, hasta el final del mes. Al menos 56.707 personas -sin contar los residentes- se encuentran en Calp durante la celebración de las fiestas de Moros y Cristianos y 95.239 personas lo hacen durante los días que dura la Oktoberfest.

En segundo lugar, y sin negar lo anterior, el estudio ha puesto de manifiesto que algunos de los impactos que se asociaban a las mismas deben ser matizados. Por un lado, el estudio ha puesto de manifiesto que ambas fiestas suponen un foco de atracción de visitantes, procedentes, sobre todo, de municipios próximos al destino. Este resultado concuerda bien con los resultados de Egresi y Kara (2014) o Getz (2007) cuando señalan que los pequeños eventos son atendidos principalmente por gente próxima a la zona. Las fiestas analizadas en el caso de Calp, ponen de relieve también que despiertan un mayor interés entre el turismo internacional -que experimenta un mejor comportamiento en términos de visitantes durante las semanas analizadas -que entre los visitantes nacionales, lo que tiene importantes implicaciones prácticas desde el punto de vista de la política de promoción de estas fiestas para los gestores turísticos del destino.

Por último, en tercer lugar, el estudio ha revelado que el análisis basado en las metodologías tradicionales, tienden a sobreestimar los impactos. En el caso de Calp, por comparación con el resultado obtenido en Perles-Ribes (2006) para el caso de la fiesta de Moros y Cristianos, dicha sobreestimación se concreta en un orden del doble.

En un plano más general, del ejercicio efectuado pueden obtenerse lecciones para otros destinos y el análisis y proyección de las festividades locales como elementos de atracción turística y desestacionalización de los flujos de demanda. En el marco de la programación, aunque muchos eventos y festividades se celebran en fechas fijas debido a la tradición, el caso de Calp demuestra que la cercanía de este tipo de eventos y fiestas a la temporada alta de afluencia turística -más que en períodos aislados en medio de la temporada baja - sirve con el tiempo a su progresiva prolongación, optimizando el potencial turístico de las mismas al permanecer toda la infraestructura turística del destino abierta para albergar al flujo de demanda generado.

En el plano del análisis, el ejercicio efectuado pone de manifiesto que, mediante las modernas técnicas de análisis basadas en la captación de datos provenientes de la telefonía móvil u otros elementos tecnológicos similares, puede obtenerse una mayor precisión en el análisis de los flujos turísticos y en la estimación de los impactos económicos generados por este tipo de eventos, por lo que respecta a su mayor o menor concentración o difusión en las dimensiones temporales o geográficas. Lo que resulta mucho más difícil, impreciso y costoso mediante las técnicas clásicas de análisis. Ahora bien, y como hemos visto en este caso, siempre que la tecnología vaya acompañada de un adecuado diseño de la investigación a llevar a cabo.

En este sentido, el estudio aquí no está exento de limitaciones, siendo la principal la dificultad extraer una interpretación causal de los resultados obtenidos. Como se ha dicho, para interpretar en términos causales los resultados obtenidos sería necesario establecer un verdadero contrafactual al fenómeno analizado, y que sería la observación de la afluencia turística al municipio durante esos mismos períodos analizados sin que la fiesta tuviera lugar. Ello no es posible con los datos de un único ejercicio y, por tanto, en este trabajo se ha tratado de aproximar -sin que pueda asumirse que se consigue completamente por las distorsiones que los puentes y festivos existentes generan en los períodos considerados de control- mediante la comparación de los resultados de los períodos objeto de análisis y unos períodos seleccionados a modo de control que se han demostrado poco útiles.

Así, un avance hacia una mejor comprensión de los impactos considerados y su interpretación causal obligaría, bien a disponer de datos longitudinales que permitieran observar, a través de las distintas variaciones que las fechas concretas de las fiestas van observando a lo largo de sus distintas ediciones, los verdaderos resultados que estas variaciones van generando, bien la consideración de un período de control -tal vez en noviembre- mejor representativo de la dinámica del destino en ausencia de las fiestas.

En cualquier caso, el estudio efectuado ilustra bien un caso de aplicación de las nuevas tecnologías al análisis económico del turismo, pone de relieve las virtudes que estas aplicaciones pueden tener para 
la mejor comprensión del fenómeno, y advierte de que la tecnología, por sí misma, no resuelve todos los problemas si no va acompañada de un diseño adecuado en el plano científico de la investigación. Todo ello representa un avance respecto a la literatura existente en la materia.

\section{Bibliografía}

Ayuntamiento de Calp 2016. Estudio Turístico de Calp 2016. www.calpe.es

Bassolas, A., Lenormand, M., Tugores, A., Gonçalves, B., Ramasco, J.J. 2016. Touristic site attractivenes seen through twitter. EPJ Data Science, 5 (1).

Birenboim, A. 2016. New approaches to study of tourist experiences in time and space. Tourism Geographies, 18 (1):9-17

Crompton, J.L., McKay,S.L. 1994. Measuring the economic impact of festivals and events: some myths, missaplications and ethical dilemmas, Festival Management \& Event Tourism, 2:33-43.

Dwyer, L., Forsyth, P., Spurr, R. 2005. Estimating the impacts of special events on an economy, Journal of Travel Research, 43:351-359.

Egresi, I., Kara, F. 2014. Economic and tourism impact of samll events: the case of small-scale festivals in Instanbul, Turkey, Studia Urb Geographia, 59, 1:47-64.

Fernández-Alcantud, A., López-Morales, J.M., Moreno-Izquierdo, L., Perles-Ribes, J. F., Ramón-Rodríguez, A. \& Such-Devesa, M.J. 2017. Innovación y destinos inteligentes: oportunidad para el know how turístico español, Información Comercial Española, 894: 137-150.

Fourie, J., Santana-Gallego, M. 2011. The impact of mega-sport events on tourist arrivals, Tourism Management, 32:1364-1370.

Frederiksen, L. 2012. Big data, Public Services Quarterly, 8 (4):345-349

Fuchs, M., Höpken, W. \& Lexhagen, M. 2014. Big data analytics for knowledge generation in tourism destinations - A case from Sweden, Journal of Destination Marketing and Management, 3 (4):198-209.

Gelan, A. 2003. Local economic impacts: the British Open, Annals of Tourism Research, 30 (2):406-425.

Getz, D. 2007. Event studies: theory, research and policy for planned events. Oxford: Butterworth-Heinemann.

Herrero-Prieto, L.C. 2010. La contribución de la cultura y las artes al desarrollo económico regional, Investigaciones Regionales, 19:177-202

IBM 2019. ¿Qué es big data?. IBM Developer. www.ibm.com (accedido 10 de julio de 2019)

Imbens, G.W., Rubin, D.B. 2015. Causal inference for statistics, social and biomedical sciences. An introduction. Cambridge University Press. New York

Jackson, J., Houghton, M., Russell, R., Triandos, P. 2005. Innovations in measuring economic impacts of regional festivals: a do-it-yourself kit, Journal of Travel Research, 43 (4): 360-367

Krom, J.C.M. 2008. Festivals of Moors and Christians: Performance, Commodity and Identitiy in Folk Celebrarions in Southern Spain, Journal of Mediterranean Studies, 18 (1):119-138.

Leng, Y., Noriega, A., Pentland, A., Winder, I., Lutz, N., Alonso, L. 2016. Analysis of Tourism Dynamics and Special Events through Mobile Phone Metadata. Bloomberg Data for Good Exchange Conference. 25-09-2016. New York City, NY. USA.

Morales-Yago, F.J., Martínez-Puche, A., Martínez-Puche, S. 2018. Las fiestas de origen histórico como instrumento para fomentar el turismo en un espacio de frontera del Sureste peninsular, Boletín de la Asociación de Geógrafos Españoles, 76:247-275.

Moscardo, G. 2007. Analyzing the role of festivals and events in regional development, Event Management, 11,1-2:23-32

Perles-Ribes, J.F. 2006. Análisis del impacto económico de eventos: una aplicación a fiestas populares de proyección turística, Cuadernos de Turismo, 17:147-166

Perles-Ribes, J. F., Ramón Rodríguez, A., Vera-Rebollo, J.F \& Ivars-Baidal, J.A. 2017. The end of growth in residential tourism destinations: steady state or sustainable development? The case of Calpe, Current Issues in Tourism, 21(12).

Perles-Ribes, J.F., Ivars-Baidal, J.A. 2018. Smart sustainability: a new perspective in the sustainable tourism debate, Investigaciones Regionales - Journal of Regional Research, 42: 151-170

Song, H., Liu, H. 2017. Predicting tourist demand using big data. In Z. Xiang, D.R.Fesenmaier (eds.), Analytics in Smart Tourism Design, Concepts and Methods. Springer International Publishing Switzerland. 
Tohmo, T. 2005. Economic impacts of cultural events on local economis: an input-output analysis of the Kaustinen Folk Music Festival, Tourism Economics, 11 (3):431-451

Wood, S.A., Guerry, A.D., Silver, J.M., Lacayo, M. 2013. Using social media to quantify nature-based tourism and recreation. Scientific reports, 3. 